\title{
Country ID:
}

Site ID:

FORM ID:

Implementation Research On Management Of Possible Serious Bacterial Infection (PSBI) In Young Infants (0-59 Days) Where Referral Is Not Feasible

\begin{tabular}{|c|c|c|c|}
\hline 1. $\mathrm{PHC}-$ & 2. Sub-centre- & 3. Village- & 4. ASHA - \\
\hline 5. Mother- & 6. Father- & 7. Religion- Hindu / Muslim / Sikh / Other & 8. Caste- SC / Other \\
\hline
\end{tabular}

\begin{tabular}{|c|c|c|c|}
\hline 9. Visit for Pregnancy preparation- & l & 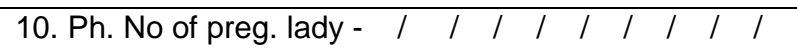 & 11.Date of admission for delivery - \\
\hline 12.Date of Delivery - & & $\begin{array}{l}\text { 13. Place of delivery - Dist. Hospital. / CHC / PHC / } \\
\text { Sub-centre / Pvt. / home / road / other /NC }\end{array}$ & 14. Type of delivery- Normal / Caesarean / assisted / NC \\
\hline 15. Time of Delivery & AM/PM & $\begin{array}{l}\text { 16. Outcome - live birth / Still birth / died after birth / NC } \\
\text { / NAS }\end{array}$ & 17. Birth weight (in grms)- \\
\hline 18.Sex of Child - Male / Female/ Other / NC & & $\begin{array}{l}\text { 19. When infant breastfed for first time- } \\
\text { First hour / first day / after } 1^{\text {st }} \text { day / did not / NC }\end{array}$ & 20. Hospital discharge date (Mother)- \\
\hline 21. Hospital discharge date (Child) - & I & $\begin{array}{l}\text { 22.Child's MCTS No; } \\
I \quad l \quad l \quad l \quad l \quad l \quad l\end{array}$ & 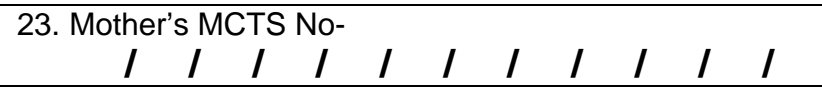 \\
\hline
\end{tabular}

(Q 25 to Q 27: TO be filled if any supervision visits were made for this infant)

\begin{tabular}{|l|l|l|} 
26.Designation of Supervisor- & 27. Date of supervision- & $I$
\end{tabular}

Description of assessment by ASHAs during HBPNC visit

\begin{tabular}{|c|c|c|c|c|c|c|c|c|c|}
\hline \multicolumn{10}{|c|}{ t. } \\
\hline Visit number & 28.Visit 1 & 29. Visit 2 & 30.Visit 3 & 31. Visit 4 & 32. Visit 5 & 33. Visit 6 & 34. Visit 7 & 35.Visit 8 & \multirow{3}{*}{$\begin{array}{l}\text { 36.Sup. Visit } \\
\text { (fill up, if visit is } \\
\text { made) }\end{array}$} \\
\hline Day after delivery & 1day & 3rd day & 7th day & 14th Day & 21st day & 28th day & 42nd day & $59^{\text {th }}$ Day & \\
\hline I. Date of visit & $I$ & $I$ & $I$ & $I$ & $I$ & $I$ & $I$ & I & \\
\hline $\begin{array}{l}\text { II. Baby alive (If response yes, skip } \\
\text { to V) }\end{array}$ & Yes / No / NC & Yes / No / NC & Yes / No / NC & Yes / No / NC & Yes / No / NC & Yes / No / NC & Yes / No / NC & Yes / No / NC & $\mathrm{R} / \mathrm{W} / \mathrm{NC}$ \\
\hline III. If Died, date of death & I & I & $I$ & I & I & 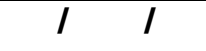 & I & I & $\mathrm{R} / \mathrm{W} / \mathrm{NC}$ \\
\hline $\begin{array}{l}\text { IV. Place of Death ( Institutional / } \\
\text { Private/ other - elaborate) }\end{array}$ & & & & & & & & & $\mathrm{R} / \mathrm{W} / \mathrm{NC}$ \\
\hline $\begin{array}{l}\text { V. Infant was clothed according to } \\
\text { weather? }\end{array}$ & Yes / No / NC & Yes / No / NC & Yes / No / NC & Yes / No / NC & Yes / No / NC & Yes / No / NC & Yes / No / NC & Yes / No / NC & $\mathrm{R} / \mathrm{W} / \mathrm{NC}$ \\
\hline VI. Bathed? & Yes / No / NC & & & & & & & & $\mathrm{R} / \mathrm{W} / \mathrm{NC}$ \\
\hline VII. Supplementary feeding? & Yes / No / NC & Yes / No / NC & Yes / No / NC & Yes / No / NC & Yes / No / NC & Yes / No / NC & Yes / No / NC & Yes / No / NC & $\mathrm{R} / \mathrm{W} / \mathrm{NC}$ \\
\hline
\end{tabular}

Version 4 


\section{Country ID:}

Site ID:

FORM ID:

Implementation Research On Management Of Possible Serious Bacterial Infection (PSBI) In Young Infants (0-59 Days) Where Referral Is Not Feasible

\begin{tabular}{|c|c|c|c|c|c|c|c|c|c|}
\hline Visit number & 28. Visit 1 & 29.Visit 2 & 30.Visit 3 & 31.Visit 4 & 32. Visit 5 & 33. Visit 6 & 34. Visit 7 & 35. Visit 8 & 36.Sup. Visit \\
\hline $\begin{array}{l}\text { VIII. Complete breast feeding ( } 5-6 \text { times } \\
\text { in day and } 3 / 4 \text { times in night) }\end{array}$ & Yes / No / NC & Yes / No / NC & Yes / No / NC & Yes / No / NC & Yes / No / NC & Yes / No / NC & Yes / No / NC & Yes / No / NC & $\mathrm{R} / \mathrm{W} / \mathrm{NC}$ \\
\hline $\begin{array}{l}\text { IX. Weight of the infant, in grams ( write } \\
9999 \text { if not documented) }\end{array}$ & & & & & & & & & $\mathrm{R} / \mathrm{W} / \mathrm{NC}$ \\
\hline $\begin{array}{l}\text { X. Temperature of the infant, in }{ }^{\circ} \mathrm{F} \\
\text { (write } 999.9 \text { if not documented) }\end{array}$ & & & & & & & & & $\mathrm{R} / \mathrm{W} / \mathrm{NC}$ \\
\hline $\begin{array}{l}\text { XI. Counted respiratory rate/min ( write } \\
99 \text { if not documented) }\end{array}$ & & & & & & & & & $\mathrm{R} / \mathrm{W} / \mathrm{NC}$ \\
\hline XII. Was umbilicus draining pus? & Yes / No / NC & Yes / No / NC & Yes / No / NC & Yes / No / NC & Yes / No / NC & Yes / No / NC & Yes / No / NC & Yes / No / NC & $\mathrm{R} / \mathrm{W} / \mathrm{NC}$ \\
\hline XIII. Was Eyes draining pus? & Yes / No / NC & Yes / No / NC & Yes / No / NC & Yes / No / NC & Yes / No / NC & Yes / No / NC & Yes / No / NC & Yes / No / NC & $\mathrm{R} / \mathrm{W} / \mathrm{NC}$ \\
\hline XIV. < 10 skin pustules? & Yes / No / NC & Yes / No / NC & Yes / No / NC & Yes / No / NC & Yes / No / NC & Yes/ No / NC & Yes / No / NC & Yes / No / NC & $\mathrm{R} / \mathrm{W} / \mathrm{NC}$ \\
\hline \multicolumn{10}{|c|}{ (If following signs were present ASHAs are mandated to inform ANM IMOs OR immediately call 102 ambulance and refer the infant to nearest health facility) } \\
\hline XV. Convulsions? & Yes / No / NC & Yes / No / NC & Yes / No/NC & Yes / No / NC & Yes / No / NC & Yes / No / NC & Yes / No / NC & Yes / No / NC & $\mathrm{R} / \mathrm{W} / \mathrm{NC}$ \\
\hline $\begin{array}{l}\text { XVI. Respiratory rate } \geq 60 \text { (Fast } \\
\text { breathing) }\end{array}$ & Yes / No / NC & Yes / No / NC & Yes / No / NC & Yes / No / NC & Yes / No / NC & Yes / No / NC & Yes / No / NC & Yes / No / NC & $\mathrm{R} / \mathrm{W} / \mathrm{NC}$ \\
\hline XVII. Chest indrawing? & Yes / No / NC & Yes / No / NC & Yes / No / NC & Yes / No / NC & Yes / No / NC & Yes / No / NC & Yes / No / NC & Yes / No / NC & $\mathrm{R} / \mathrm{W} / \mathrm{NC}$ \\
\hline $\begin{array}{l}\text { XVIII. Stopped feeding/ unable to feed } \\
\text { well? }\end{array}$ & Yes / No / NC & Yes / No / NC & Yes / No / NC & Yes / No / NC & Yes / No / NC & Yes/ No/NC & Yes / No / NC & Yes / No / NC & $\mathrm{R} / \mathrm{W} / \mathrm{NC}$ \\
\hline XIX. Body temp. $\geq 99^{\circ}$ F OR $<95^{\circ} \mathrm{F}$ & Yes / No / NC & Yes / No / NC & Yes / No / NC & Yes / No / NC & Yes / No / NC & Yes / No / NC & Yes / No / NC & Yes / No / NC & $\mathrm{R} / \mathrm{W} / \mathrm{NC}$ \\
\hline $\begin{array}{l}\text { XX. Movement on simulation/ No } \\
\text { movement / less movement }\end{array}$ & Yes / No / NC & Yes / No / NC & Yes / No / NC & Yes / No / NC & Yes / No / NC & Yes / No / NC & Yes / No / NC & Yes / No / NC & $\mathrm{R} / \mathrm{W} / \mathrm{NC}$ \\
\hline \multirow[t]{2}{*}{ XXI. Low body weight } & \multicolumn{2}{|c|}{$<\frac{1}{<2000 \mathrm{gms}}$} & $<2100 \mathrm{gms}$ & $<2100 \mathrm{gms}$ & $<2200 \mathrm{gms}$ & $<2300 \mathrm{gms}$ & $<2500 \mathrm{gms}$ & $<2500 \mathrm{gms}$ & $<2500 \mathrm{gms}$ \\
\hline & Yes / No / NC & Yes/No/NC & Yes / No / NC & Yes / No / NC & Yes / No / NC & Yes/ No/NC & Yes / No / NC & Yes / No / NC & $\mathrm{R} / \mathrm{W} / \mathrm{NC}$ \\
\hline XXII. $\geq 10$ Skin pustules Or a big Boil & Yes / No / NC & Yes / No / NC & Yes / No / NC & Yes / No / NC & Yes / No / NC & Yes / No / NC & Yes / No / NC & Yes / No / NC & $\mathrm{R} / \mathrm{W} / \mathrm{NC}$ \\
\hline XXIII. Palms/soles/ body yellow? & Yes / No / NC & Yes / No / NC & Yes / No / NC & Yes / No / NC & Yes / No / NC & Yes / No / NC & Yes / No / NC & Yes / No / NC & $\mathrm{R} / \mathrm{W} / \mathrm{NC}$ \\
\hline XXIV. Lips/ face/ Body became blue & Yes / No / NC & Yes / No / NC & Yes / No / NC & Yes / No / NC & Yes / No / NC & Yes / No / NC & Yes / No / NC & Yes / No / NC & $\mathrm{R} / \mathrm{W} / \mathrm{NC}$ \\
\hline XXV. Umbilicus bleeding & Yes / No / NC & Yes / No / NC & Yes / No / NC & Yes / No / NC & Yes / No / NC & Yes / No / NC & Yes / No / NC & Yes / No / NC & $\mathrm{R} / \mathrm{W} / \mathrm{NC}$ \\
\hline XXVI. Did not urinate within $24 \mathrm{hrs}$ of birth & Yes / No / NC & & & & & & & & $\mathrm{R} / \mathrm{W} / \mathrm{NC}$ \\
\hline $\begin{array}{l}\text { KXVII. Did not passed stool within } 48 \text { hrs of } \\
\text { birth }\end{array}$ & Yes / No / NC & Yes / No / NC & & & & & & & $\mathrm{R} / \mathrm{W} / \mathrm{NC}$ \\
\hline XVIII. Blood in stool & Yes / No / NC & Yes / No / NC & Yes / No / NC & Yes / No / NC & Yes / No / NC & Yes / No / NC & Yes / No / NC & Yes / No / NC & $\mathrm{R} / \mathrm{W} / \mathrm{NC}$ \\
\hline $\begin{array}{l}\text { XXIX. Watery diaherroea more than } 3 \\
\text { times in a day with symptoms of } \\
\text { dehydration }\end{array}$ & Yes / No / NC & Yes / No / NC & Yes / No / NC & Yes / No / NC & Yes / No / NC & Yes / No / NC & Yes / No / NC & Yes / No / NC & $\mathrm{R} / \mathrm{W} / \mathrm{NC}$ \\
\hline
\end{tabular}

Version 4 


\section{Country ID:}

Site ID:

FORM ID:

Implementation Research On Management Of Possible Serious Bacterial Infection (PSBI) In Young Infants (0-59 Days) Where Referral Is Not Feasible

Fill this section if the infant is referred By ASHA and the information is documented in HBPNC form

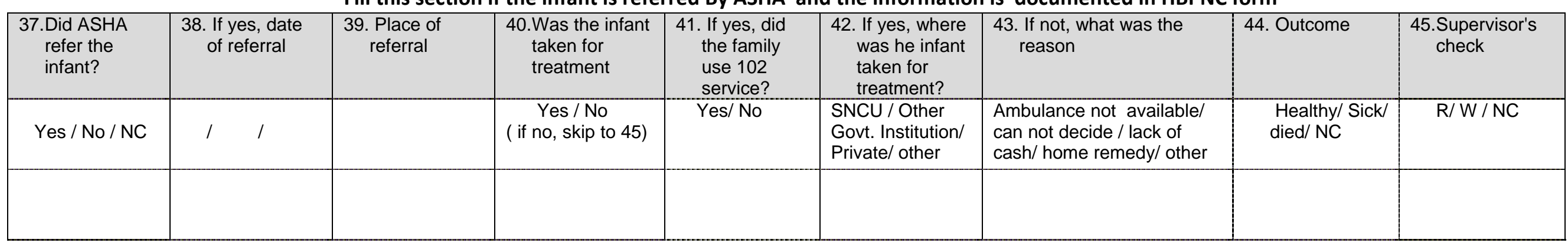

\section{Verification of ASHA's HBNC Visits}

\begin{tabular}{|c|c|c|c|c|c|c|c|c|c|c|c|}
\hline & Verification & $\begin{array}{l}\text { 46. Pre delivery } \\
\text { visit } \\
\text { Verification }\end{array}$ & $\begin{array}{l}\text { 47.Day } 1 \\
\text { Verification }\end{array}$ & $\begin{array}{l}\text { 48.Day } 3 \\
\text { Verification }\end{array}$ & $\begin{array}{l}\text { 49.Day } 7 \\
\text { Verification }\end{array}$ & $\begin{array}{l}\text { 50.Day } 14 \\
\text { Verification }\end{array}$ & $\begin{array}{l}\text { 51.Day } 21 \\
\text { Verification }\end{array}$ & $\begin{array}{l}\text { 52. Day } 28 \\
\text { Verification }\end{array}$ & $\begin{array}{l}\text { 53.Day } 42 \\
\text { Verification }\end{array}$ & $\begin{array}{l}\text { 54.Day } 59 \\
\text { Verification }\end{array}$ & $\begin{array}{l}\text { 55. Supervisor's } \\
\text { check }\end{array}$ \\
\hline $\mathrm{I}$. & Signed by Mother & Yes / No & Yes / No & Yes / No & Yes / No & Yes / No & Yes / No & Yes / No & Yes / No & Yes / No & \multirow{2}{*}{$\mathrm{R} / \mathrm{W} / \mathrm{NC}$} \\
\hline II. & $\begin{array}{l}\text { Date of verification by mother } \\
\text { ( write } 99 / 99 / 99 \text { if no date) }\end{array}$ & $1 \quad 1$ & I & $1 \quad 1$ & I & I & 1 & I & I & I & \\
\hline III. & Signed by ASHA supervisor & Yes / No & Yes / No & Yes / No & Yes / No & Yes / No & Yes / No & Yes / No & Yes / No & Yes / No & \\
\hline IV. & $\begin{array}{l}\text { Date of verification by ASHA } \\
\text { supervisor } \\
\text { ( write } 99 / 99 / 99 \text { if no date) }\end{array}$ & 11 & I & I & I & l & I & l & I & l & \\
\hline
\end{tabular}

56.Migrated - Yes / No 57.Date of Migration-

\section{Fill this section if the Family moved away}

58.Place of Migration (ADDRESS)-

59. Name of the $\mathrm{HH}$ head (where migrated)-

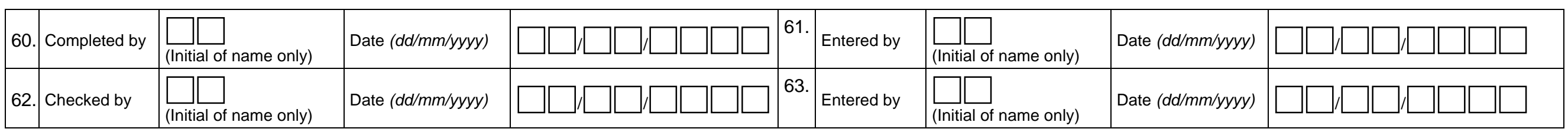

Version 4 